\title{
Watermarking Road Maps against Crop and Merge Attacks
}

\author{
Kai Jiang Kenny Q. Zhu \\ Shanghai Jiao Tong University \\ Shanghai, China \\ jkai@sjtu.edu.cn, \\ kzhu@cs.sjtu.edu.cn
}

\author{
Yan Huang \\ University of North Texas \\ Denton, TX, USA \\ huangyan@unt.edu
}

\author{
Xiaobin $\mathrm{Ma}$ \\ 1 Oracle Drive \\ Nashua, NH, USA \\ xiaobin@cs.umn.edu
}

\begin{abstract}
Past research on watermarking digital road maps has been focused on deterring common attacks such as adding noise to the whole map so as to destroy the embedded watermarks. This paper focuses on two less common but increasingly used types of attack: crop attacks and merge attacks. Crop attack crops a fragment of the original map and uses the fragment as a new map. When the new map is much smaller than the original map, it is called massive cropping. Merge attack merges maps from various sources together to form a new map. Conventional watermarking techniques fail against these attacks either because they require global information from the whole map or they must add too many local watermarks and affect the usability of the maps. This paper proposes a novel quad-tree based blind watermarking scheme that partitions the original map according to the quad-tree and plants just one single bit in each sub-region of the map. The approach achieves almost 100\% detection accuracy for moderate crop and merge attacks, and over $80 \%$ accuracy with more than $95 \%$ of the original map cropped and removed. Furthermore, the method introduces very little distortion to the original map: to effectively protect a $23.5 \mathrm{MB}$ MinneapolisSt.Paul map against crop and merge as well as other common attacks, only 423 bits or 53 bytes of watermark is required. ${ }^{1}$
\end{abstract}

\section{Categories and Subject Descriptors}

D.2.11 [Software Architectures]: Information hiding; D.4.6 [Security and Protection]: Authentication

\section{Keywords}

Watermark, digital road map, quad-tree, crop attack, merge attack

\section{INTRODUCTION}

Digital watermarking is an important technique to protect the copyrights of digital products such as images, audios and videos. A watermark is small amount of digital noise embedded into the digital representation of the products. Watermarking is different from

\footnotetext{
${ }^{1}$ Kenny Q. Zhu (the corresponding author) is partially supported by NSFC grants 61033002 and 61100050.
}

Permission to make digital or hard copies of all or part of this work for personal or classroom use is granted without fee provided that copies are not made or distributed for profit or commercial advantage and that copies bear this notice and the full citation on the first page. To copy otherwise, to republish, to post on servers or to redistribute to lists, requires prior specific permission and/or a fee.

IH\&MMSec'13, June 17-19, 2013, Montpellier, France.

Copyright 2013 ACM 978-1-4503-2081-8/13/06 ...\$15.00. encryption, another method used to protect digital content from unauthorized access. Watermarks embedded in a product should not be perceived by the user or the application and hence don't affect the normal use of the product, whereas encrypted product cannot be used unless the user has the means to decrypt the product, usually with the help of a secret key. Once the content is decrypted, unlimited illegal copies of the product can be made and used as if they were legal copies. Digital watermarking complements encryption. By embedding some watermarks that are hard to remove, one can always claim the ownership of the product after detecting the watermarks.

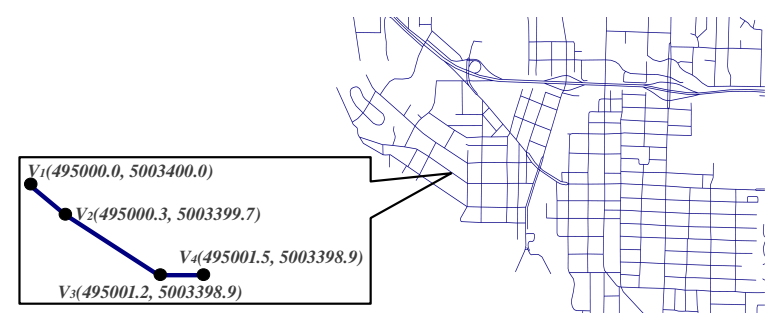

Figure 1: A Digital Road Map for Part of Anoka County, MN

In this paper, we are concerned with the protection of copyrights of digital road maps by watermarking. A digital road map is a vector graph representation of roads in a geographical region. Such maps are widely used in Geographic Positioning Systems (GPS) and other GIS or location-based applications. Figure 1 shows an example of a snippet of a road map of Anoka County, MN in the United States. In this map, a road is represented by a polyline, shown in the blow-up image, where a polyline is a sequence of connected straight line segments. Each line segment is presented digitally by its two end vertexes in terms of $(x, y)$ coordinates, where $x$ and $y$ are latitude and longitude of the point on earth, respectively. Very wide two-way roads (e.g., freeways) with central dividers are represented by two (often) parallel polylines, which are also shown in Figure 1.

The standard watermarking framework for digital road maps (shown in Figure 2) also adopts a two-step approach. The two key modules in the framework are the watermark insertion and detection algorithms. These two modules typically share some common secret keys which are only known to the algorithms. Some detection algorithms require the original map while others don't. When digital road maps are unlawfully copied for commercial use, they often undergo various modifications or transformations in hope that the original watermarks are either removed or become undetectable. Such modifications to the map is called attacks on the watermarks. Existing attacks include attempts to remove or alter the watermarks, 


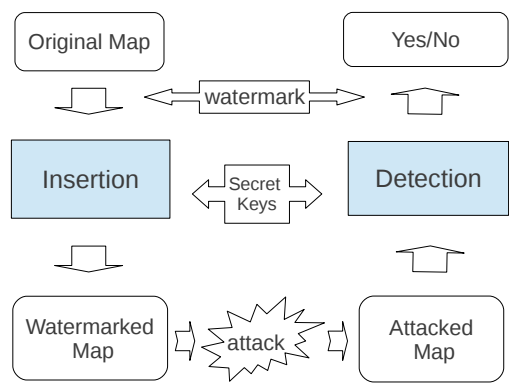

Figure 2: General Watermarking Framework For Road Maps

adding more noises to the map, cutting a map into smaller pieces or merging multiple maps from different sources. The last two attacks, which we call "crop attack" and "merge attack" are less common, and they are the focus of our research in this paper.

A scenario of a crop attack goes like this: an attacker obtains a big map, e.g., the map of the state of Minnesota, and crops out the Minneapolis - St.Paul area to create a "new" twin-city map. In the case of a merge attack, the attacker extracts maps for various counties in Minnesota from several different digital maps and composes a new Minnesota map by aligning these sub-maps properly together. The first attack is easier to implement, while the second attack is more difficult to carry out in practice and harder to defeat.

Existing watermarking techniques come in two categories, global watermarking and local watermarking. In global watermarking, the insertion module computes an overall watermark based on the global information of the data, and inserts this watermark over the whole data. Detection requires the reconstruction of the original watermark which requires global information as well. As such, these techniques cannot handle crop or merge attacks.

In local watermarking, the insertion module generates many watermarks for different regions of the data, and each watermark is computed from the local information of the corresponding regions. For example, one can partition a map into many smaller regions, and insert a watermark according to the properties of the data in each region. Local road map watermarking schemes [15, 16, 23] have the potential of surviving crop attacks. However, to handle "massive cropping", i.e., cropping out a very small piece of the original map, existing local schemes have to resort to fine-grained partitioning and the insertion of many more watermarks which may affect the perception of the map. Moreover, many of these techniques require coordination between the detection of watermarks in adjacent sub-regions (e.g., some repetitive patterns) to conclude the authenticity of the overall map. Such coordination may fail if it happens at the boundary of the cropping. Such massive cropping attacks are real, since in our previous example, the Twin-Cities area is much smaller than the whole state of Minnesota.

None of the existing local methods can defeat the merge attack because all of them compute a global confidence score based on the watermarks extracted from individual sub-regions. If a significant part of the map comes from a foreign source without the inserted watermarks, these methods typically give a low confidence score and fail to identify part of the map as being authentic. Without a proper data structure, these methods also suffer from high computation complexity if they attempt to "guess" where the watermarked region is in the map.

In this paper, we propose a novel local watermarking approach which partitions the original map by a modified quad-tree structure. The depth of the tree is determined by the road density in the region. We compute the local watermark for each partition by the total length of the roads in that partition and each watermark is represented by one bit change in the original data. During detection phase, our approach can reconstruct the quad-tree and identify the potential locations of the embedded watermarks, and can report with high confidence if a given attacked map (be it traditional attacks or crop/merge attacks) contains a sub-region which carries the original watermarks as well as reporting that sub-region itself. In addition, the detection method in our approach doesn't require the original map, but only needs a "secret grid boundary" which serves as the key. The size of this key is negligible compared to the original data. Watermark detection scheme without the need for original data is also known as "blind watermarking."

This paper makes the following key contributions:

- The framework defeats massive cropping and merging attacks with high accuracy. Our experiments show that our approach achieves $100 \%$ detection accuracy for moderate cropping and more than $80 \%$ accuracy with more than $95 \%$ of the original map cropped, whereas the accuracy of two other state-of-the-art approaches degrades to around $20 \%$ with similar massive cropping. Our method outperforms the peers by similar margins under merge attacks.

- The framework incurs little distortion. The map is partitioned into sub-regions of various sizes on demand. Watermarking position is calculated by information inside these sub-regions. We use a one-bit watermark to represent that the sub-region is watermarked. Consequently, to effectively watermark the whole Twin-Cities 7-counties map (23.5MB), our approach merely modified 423 bits.

- The framework is lightweight. It is a blind watermarking method which requires no original map for watermark detection, and the time complexity of both the insertion and detection algorithm is $O(|V| \log \mathcal{L})$ where $|V|$ is the number of vertices in the map and $\mathcal{L}$ is the total length of roads in the map.

The remainder of this paper is organized as follows. Section 2 introduces some preliminaries about the watermarking GIS digital data and formalizes the problems of interest. Section 3 discusses the proposed digital watermarking approach in detail. Section 4 describes the experiment setup and presents evaluation results on a real digital map data set. Section 5 discusses the related work, and we conclude the paper with some further work in section 6 .

\section{PROBLEM DEFINITION}

In this section, we first define digital road maps and distortions to the map. We then give the problem definition of watermarking digital road maps in terms of the interfaces of two functions, insertion and detection. Finally we describe some common attacks on digital maps with special emphasis on the two difficult attacks: crop and merge attacks.

\subsection{Digital Road Map and Its Distortions}

A digital road map, $M$, is a view of a graph $G$ with a set of vertices $V$ and a set of edges $E$. $M$ consists of a set of $k$ roads $R_{i}$, where each road is a polyline represented by a sequence of vertices in the form of $(x, y)$ coordinates in a geographical coordinate 
system (e.g., latitudes and longitudes). Formally,

$$
\begin{aligned}
G= & \{V, E\} \\
M= & \bigcup_{i=1}^{k} R_{i}, \text { where } k \geq 1 ; \text { and } \\
R_{i}= & {\left[V_{1}, V_{2}, \ldots, V_{m}\right] } \\
& \text { where } m \leq|V| ; \forall i \neq j: V_{i} \neq V_{j} ; \\
& \forall i \in[1, m]:\left(V_{i}, V_{i+1}\right) \in E ; \text { and } \\
& \forall i \in[1, m]: V_{i}=\left(x_{i}, y_{i}\right) .
\end{aligned}
$$

Whether we are watermarking or attacking a given map, we are essentially changing the original map, or adding distortion. In order to maintain usability of the map, the amount of distortion must not be larger than a threshold $\eta$ known as perception tolerance. We consider three types of distortion one can make to a map: perturb some vertices, insert some new vertices into a road, or delete some vertices from a road.

Given a road $R$, and a changed road $R^{\prime}$, let $P$ be the set of nodes in $R$ which are perturbed, and $I$ be the set of nodes in $R^{\prime}$ which are newly inserted, and $D$ be the set in $R$ of nodes which are deleted, the distortion between $R$ and $R^{\prime}$ is defined as

$$
\delta\left(R, R^{\prime}\right)=\delta(P)+\delta(I)+\delta(D)
$$

where

$$
\begin{aligned}
\delta(P) & =\sum_{V_{i} \in P}\left\|V_{i}^{\prime}-V_{i}\right\| \\
\delta(I) & =\sum_{V_{i} \in I} d\left(V_{i}, R\right) \\
\delta(D) & =\sum_{V_{i} \in D} d\left(V_{i}, R^{\prime}\right)
\end{aligned}
$$

and

$$
d\left(V_{i}, R\right)= \begin{cases}\left\|V_{i}-V_{i+1}\right\| & \text { if } i=1,\left\{V_{i}, V_{i+1}\right\} \subseteq R \\ \left\|V_{i}-V_{i-1}\right\| & \text { if } i=|R|,\left\{V_{i}, V_{i-1}\right\} \subseteq R \\ \|\boldsymbol{\alpha}\| \sin \langle\boldsymbol{\alpha}, \boldsymbol{\beta}\rangle \mid & \text { if }\left\{V_{i-1}, V_{i}, V_{i+1}\right\} \subseteq R\end{cases}
$$

where $\boldsymbol{\alpha}=V_{i}-V_{i-1}$ and $\boldsymbol{\beta}=V_{i+1}-V_{i-1}$, and $\langle\boldsymbol{\alpha}, \boldsymbol{\beta}\rangle$ means the angle of intersection between vectors $\boldsymbol{\alpha}$ and $\boldsymbol{\beta}$. Here $d\left(V_{i}, R\right)$ (see Figure 3 ) means the distance between a node $V_{i}$ and the line segment $\left(V_{i-1}, V_{i+1}\right)$, or if $V_{i}$ happens to be the end of a road, the distance to its adjacent node in the road.

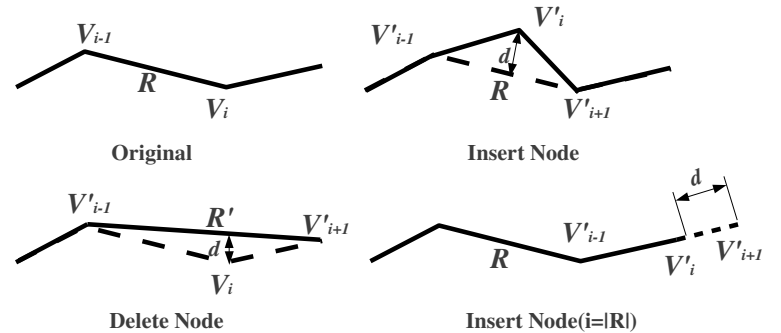

Figure 3: Distance Measurement function $d(V, R)$

The distortion to the whole map is hence the total distortion to all its roads, normalized by the total lengths of all roads:

$$
\delta\left(M, M^{\prime}\right)=\frac{\sum_{R \in M, R^{\prime} \in M^{\prime}} \delta\left(R, R^{\prime}\right)}{\sum_{R \in M} \text { length }(R)} .
$$

In the remainder of this paper, we use $\delta_{w}$ to represent the $\delta\left(M, M^{\prime}\right)$ caused by watermarking, and use $\delta_{n}$ for $\delta\left(M, M^{\prime}\right)$ due to noise.

\subsection{Insertion and Detection of Watermarks}

For a digital road map $M$, watermark $W$, and some secret key $K$ (to enhance robustness against attacks), the interface of watermarking process can be represented by two functions $\mathcal{I}$ and $\mathcal{D}$.

$$
\begin{aligned}
& \mathcal{I} \quad: \quad(M, W, K) \rightarrow M^{\prime} \\
& \mathcal{D}: \quad(M, W, K) \rightarrow\{T \mid F\}
\end{aligned}
$$

where $M^{\prime}$ is the watermarked map, $\delta\left(M, M^{\prime}\right) \leq \eta$, and detection process returns a boolean value of either true or false.

\subsection{Attacks}

There are many different attacks on digital road map. In this section We focus on into three types: the first type is a common attack which introduces random noises into an illegal copy of the map. The other two types are more complex attacks which involves cropping a given map into smaller pieces and merging pieces together from various sources.

\section{Noise Attack}

An attacker disturbs the watermarks in a map by selecting a subset of the nodes in the map and perturb the position of these nodes slightly. The subset could be small compared to the whole map:

$$
\operatorname{noise}(M)=\left\{\operatorname{noise}(R) \mid R \in M_{1}\right\} \cup M_{2}
$$

where $M_{1} \cap M_{2}=\emptyset, M_{1} \cup M_{2}=M, \operatorname{noise}(R)=[\operatorname{perturb}(V) \mid V \in$ $R]$, where $[f(V) \mid V \in R]$ a list comprehension constructed from another list $R$.

\section{Crop Attack}

An attacker crop a geographical region from the watermarked map and use it as if it's a new map. The crop attack can defeat almost all global watermarking techniques because the global information can be destroyed by the cropping. Even for many local watermarking techniques, the resistance against cropping is limited if just a small piece of the map is cropped. We define cropping attack as:

$$
\operatorname{Crop}(M)=\left\{\operatorname{subseg}(R) \mid R \in M^{\prime}\right\}
$$

where $M^{\prime} \subseteq M$ and $\operatorname{subseq}(R)$ returns a subsequence of road $R$.

If $M^{\prime}$ is a very small subset of $M$, we call the attack "massive crop" attack. None of the existing watermarking approaches can handle massive crop attack.

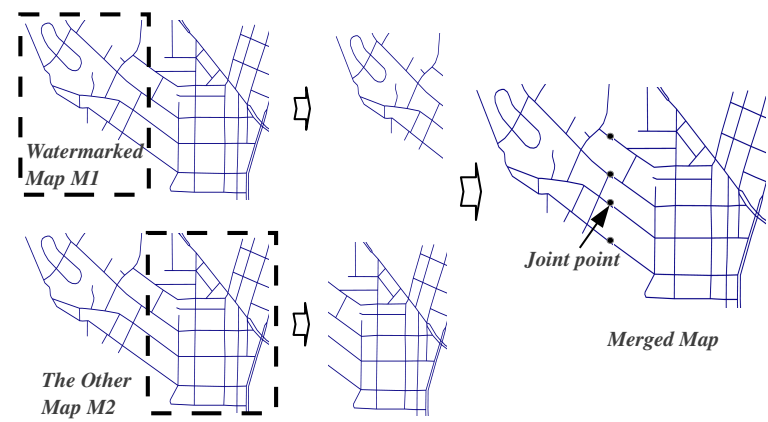

Figure 4: Merge Attack

\section{Merge Attack}

This attack involves the merging of multiple maps from different sources (see Figure 4). Maps from various sources differ by either precision of measurement, the granularity of road segmentation, or 
minor details of roads due to changes to the road over time. However, maps of the same region will be largely identical. This enables the attacker to crop parts from different maps and piece them together to make a new map. There might some slight distortion or inaccuracy at the boundaries but the resulting map is usable. We define the "merge" of two maps as:

$$
\operatorname{Merge}\left(M_{1}, M_{2}\right)=M_{\text {sep }} \cup M_{\text {con }}
$$

where

$$
\begin{aligned}
& M_{\text {sep }}=\left\{R_{1} \mid R_{1} \in M_{1}\right. \text { and } \\
& \cup\left.\forall R_{2} \in M_{2}: \operatorname{connect}\left(R_{1}, R_{2}\right)=\text { false }\right\} \\
&\left\{R_{2} \mid R_{2} \in M_{2}\right. \text { and } \\
&\left.\forall R_{1} \in M_{1}: \operatorname{connect}\left(R_{1}, R_{2}\right)=\text { false }\right\} \\
& M_{\text {con }}=\left\{\text { join }\left(R_{1}, R_{2}\right) \mid \forall R_{1} \in M_{1}, R_{2} \in M_{2},\right. \\
&\left.\operatorname{connect}\left(R_{1}, R_{2}\right)=\text { true }\right\}
\end{aligned}
$$

and connect $\left(R_{1}, R_{2}\right)$ is a boolean function that evaluates to true if two roads $R_{1}$ and $R_{2}$ share a name and an endpoint. join $\left(R_{1}, R_{2}\right)$ returns a road that joins two connected roads with identical name. $M_{\text {sep }}$ and $M_{\text {con }}$ are two sets of roads that make up the merged map, where $M_{\text {sep }}$ denotes those roads from the original maps which are disjoint and $M_{\text {con }}$ denotes a set of roads from the boundaries of the two input maps which are joined together.

\section{OUR APPROACH}

In this section, we propose a novel watermarking approach, which can effectively survive "massive crop" and "merge" attacks. Our watermarking method inserts negligible watermarks (one-bit only) to locations which are determined by a spatial partitioning algorithm. Then during detection time, the same spatial partitioning algorithm is used with the help of a secret key, which ensures that the resulting segmentation of the space and the input map is almost identical to that of the watermarked map, even though no original map is available. In the following, we first introduce the secret keys used in this framework, then present the space partitioning algorithm, watermark insertion and detection algorithms, before giving analysis of the algorithms. All symbols used in the algorithms are summarized in Table 1.

Table 1: All Symbols Used in Algorithms

\begin{tabular}{|c|c||c|c|}
\hline Symbol & Definition & Symbol & Definition \\
\hline \hline$G$ & master grid & $P O$ & sub-region list \\
\hline$M$ & original data-set & $T$ & MQtree \\
\hline $\mathcal{R}$ & partitioned region & $l$ & secret square size \\
\hline$\theta$ & road length threshold & $k$ & secret hash key \\
\hline
\end{tabular}

\subsection{Secret Keys}

The secret keys, often randomly generated, are used to decide where to insert the watermarks. In the proposed algorithm, we use three secret keys: a master grid, a secret minimum bounding rectangle (MBR) and a secret square size.

The master grid $G$ is a secret coordinate system which is only known to the map producer. The grid has an origin which is certain position on earth with precise latitude and longitude, and it has a step size which defines the granularity of the grid (see Figure 5):

$$
G=\left\{\operatorname{Origin}\left(x_{0}, y_{0}\right), \text { Step }\right\}
$$

Given an original map to watermark, it can be laid out on the master grid, according to the coordinates of the vertices in the map. The secret $M B R$ is then the smallest rectangle which coincides with the grid lines and completely encloses the whole map. The red rectangle in Figure 5 is one such secret MBR of the blue road map. In our algorithm, we partition the space according to a modified Quadtree. The MBR of the map serves as an initial of the partition process, which will improve the robustness of our algorithm.

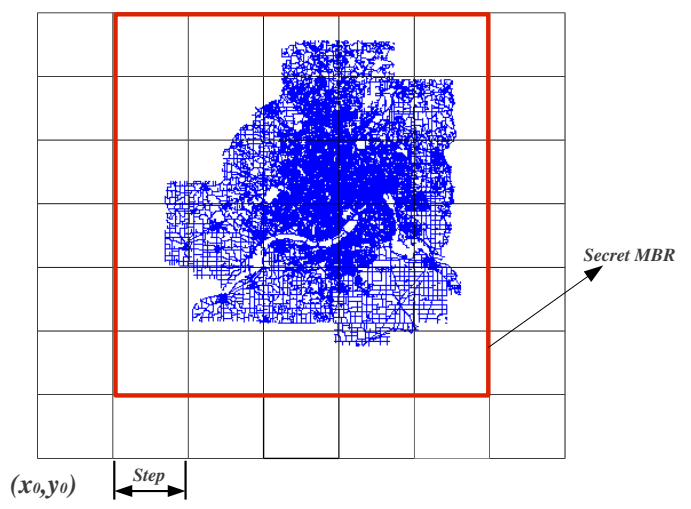

Figure 5: Master Grid and MBR

The secret square size is an integer number $l$ that determines the size of a square box that we used to select a small neighborhood of road segments from which to compute the watermarks. More details of the use of $l$ will be presented in Section 3.3.

\subsection{Space Partitioning Algorithm}

Algorithm 1 is used in both insertion and detection of the watermarks. It recursively partitions the space bounded by the secret MBR for a map into small regions using a modified quad-tree structure (known as MQtree) according to the density of the roads. Each node in MQtree represents a sub-region of the map. A leaf node represents a region in which the total length of road segments within is between $\theta$ and $4 \theta$ meters. Figure 6(b) illustrate a MQtree created from partitioning an MBR shown in Figure 6(a).

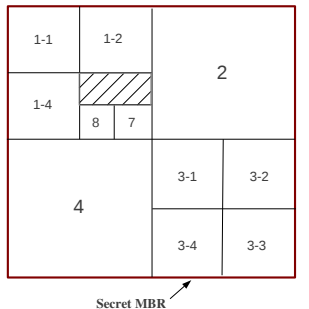

(a) Map

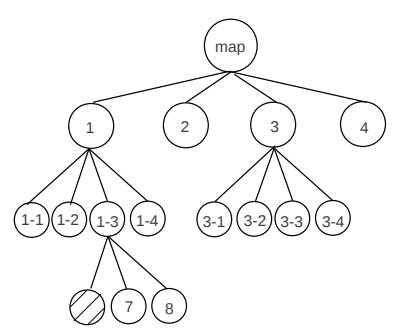

(b) MQtree

\section{Figure 6: MQtree}

In Algorithm 1, the secret MBR is generated from the master grid(see Figure 5). Then the map area is partitioned iteratively according to the density of roads such that finally the total road length included in every subregion has more than $\mathrm{m}$ meters and less than $4 * \theta$ meters. Finally, we output all of these subregions. Here we modify Quadtree by merging one subregion with less roads with its smallest neighbour when the road length of it is less than $\theta$. Road length of an area cannot be changed arbitrarily due to the constraints of perception tolerance which must be observed by both the watermark producer and the attacker. No matter a watermarked 
map is cropped or merged with other parts from different maps, this method still produces almost the identical partition results for the watermarked part.

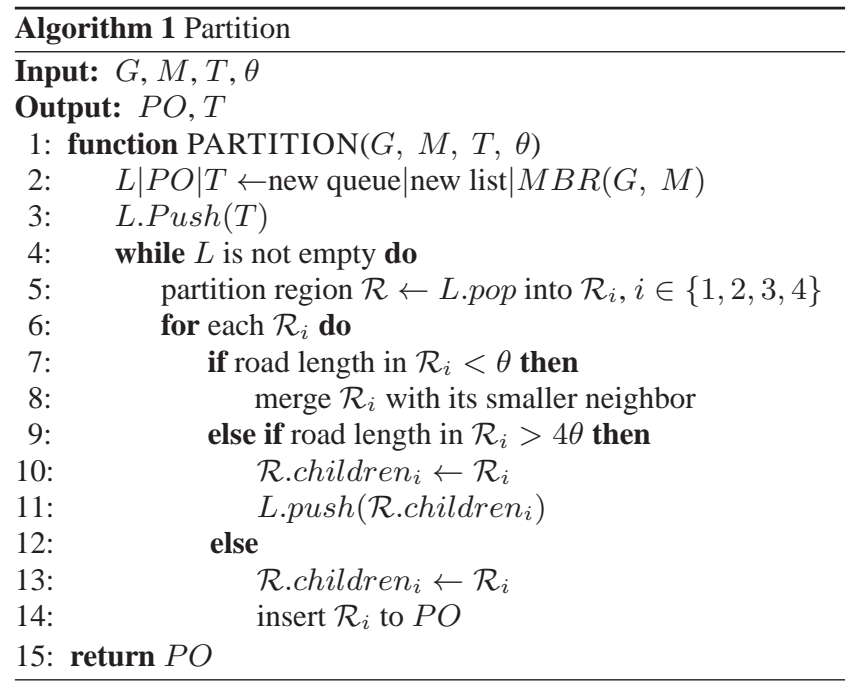

\subsection{Watermark Insertion Algorithm}

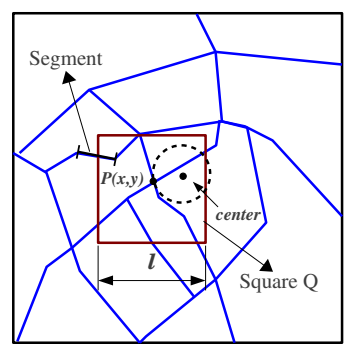

Figure 7: Insertion Strategy

Algorithm 2 first generates a set of sub-regions by partitioning. Then it inserts one watermark into each sub-region at the leaf nodes of the MQtree. The watermark is inserted into a road vertex $P(x, y)$ which is closest to the center of the sub-region (marked by the black box in Figure 7). To compute the exact watermark to be inserted, the algorithm then draw a square box of size $l$ (the other secret key) centered at $P(x, y)$ (the red box in Figure 7). Let $s l$ be the length of road segments that intersects the square box, and let $j$ be a value hashed from $s l$. We then set the $j^{\text {th }}$ least significant bit in $x$ coordinate of $P$ to " 1 ". The hash function in this algorithm must guarantee to hash to the same value before and after watermarking. Even if the watermarked map is attacked (i.e., some end points of those segments intersecting $Q$ are altered), the hash function must still hash to the same $j$. To this end, we design this function with a tolerance to possible distortions. Assuming that the biggest possible distortion of a single end point is the change of $J_{\max }$ least significant bits of the coordinates, then

$$
\begin{aligned}
& j=\operatorname{Hash}(\operatorname{Trans}(s l), k) \\
& \text { where } \operatorname{Trans}(x)=x \wedge \underbrace{11 \ldots 1 \overbrace{00 \ldots 0}^{J_{\max } \ldots}}_{\text {bit_length }(x)}
\end{aligned}
$$

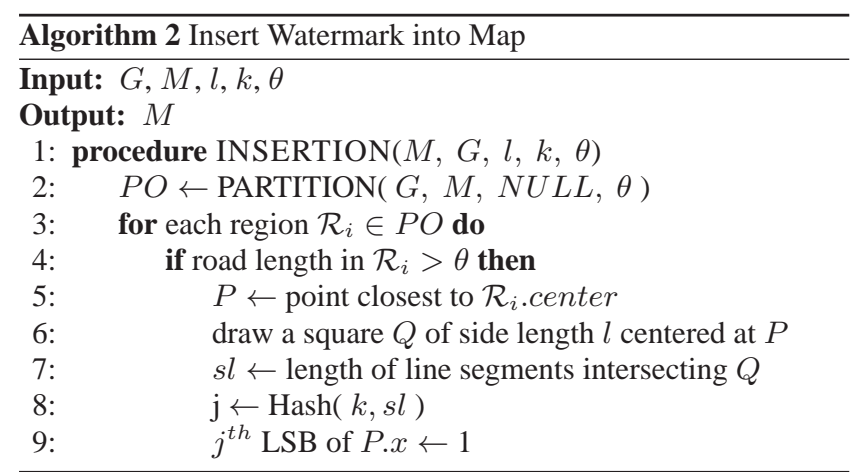

\subsection{Watermark Detection Algorithm}

In Algorithm 3, we partition the map using the same strategy as insertion. Then we select the data points closest to the center of the regions to detect whether the watermark exists there. Each subregion which is detected to contain a watermark casts a vote which collectively contributes to the final decision of whether a larger area is watermarked as a whole. For leaf nodes of the MQtree, if the bit value at the right position is " 1 ", we mark this sub-region as a "match". Function STATS(T) (Algorithm 4) computes two statistics for each node of the MQtree: the total number leaf nodes underneath the node (T.total) and the total number of nodes which has been marked as "match" ( $T$.match). If the detection confidence $\operatorname{con} f\left(T_{i}\right)$ is larger than a threshold $\xi$ for for any non-leaf node $T_{i}$, the watermark in the map is successfully identified. We define the detection confidence of $T$ as

$$
\operatorname{con} f(T)=1-\sum_{i=n}^{N}\left(\begin{array}{c}
N \\
i
\end{array}\right)\left(\frac{1}{2}\right)^{N-i}\left(\frac{1}{2}\right)^{i}
$$

where $N$ is $T$.total and $n$ is $T$.match. In our approach, the data points where we select to insert watermark may already contain " 1 " at the specified bit position. Here we assume uniform distribution and hence the probability of " 1 " being already present is $1 / 2$.

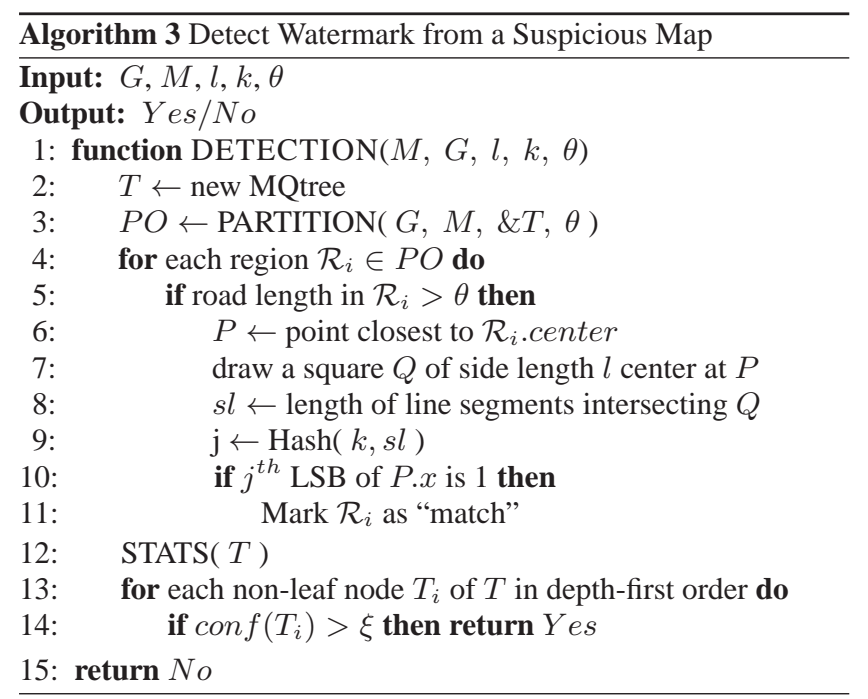

If the watermarked map is attacked by "massive crop" attack, the MQtree structure partitioned by detection algorithm is just a part of the insertion MQtree. However, it will be almost the same as the corresponding part of the MQtree for the whole map. An example of the detection process is illustrated in Figure 8. Assuming that 


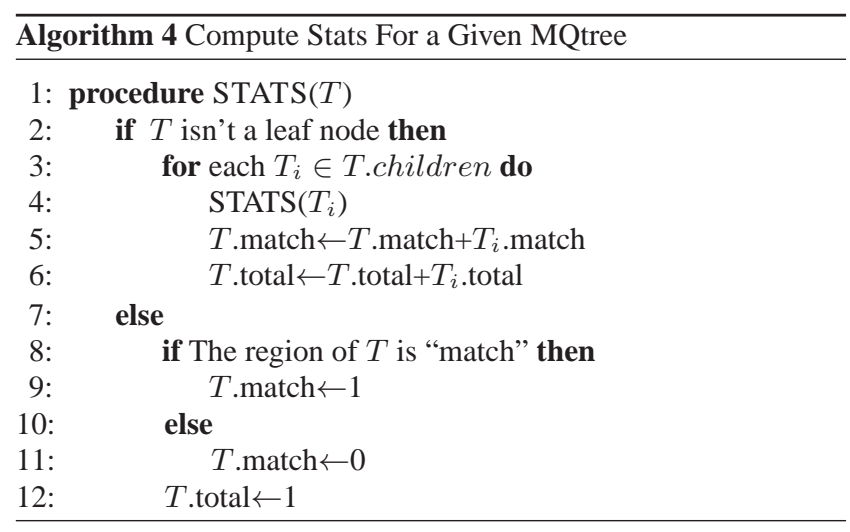

the shaded parts of Figure 8(a) is cropped from the watermarked map, the corresponding MQtree is shown in Figure 8(b).

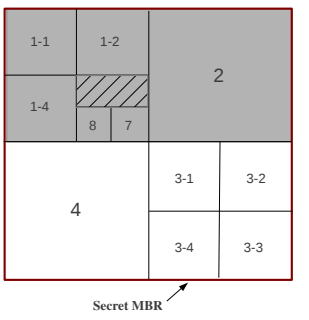

(a) Map

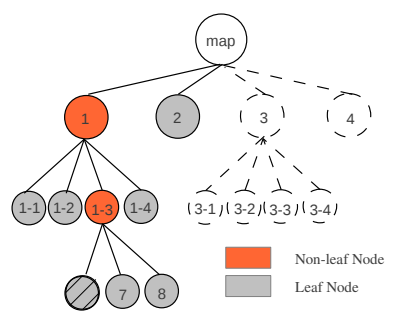

(b) MQtree
Figure 8: Detection Strategy

If the watermarked map is attacked by "Merge Attack", the detection MQtree will be almost the same as the insertion one. However, we can find the watermark only in part of the MQtree. In this case, the algorithm reports the sub-regions that are watermarked. We can make a depth-first traversal of the detection MQtree to find the largest watermarked region.

\subsection{Analysis}

The time complexity of Algorithm 1 is

$$
\mathcal{T}=O(|E| \log \mathcal{L})
$$

where $\mathcal{L}$ is the total length of all roads in map $M$. Since the number of edges connected to a vertex is bounded a small number $q$, that is, $|E| \leq(q \times|V|) / 2, \mathcal{T}=O(|V| \log \mathcal{L})$.

The time complexity of Algorithm 2 and Algorithm 3 is $\mathcal{T}+$ $O(|V|)$, or $O(|V| \log \mathcal{L})$.

The detection confidence in (1) essentially represents the probability of correctly identifying watermarks in a given map (attacked or not). We now attempt to give a lower bound to the detection confidence. Intuitively, as $\theta$ decreases, the map is divided into more subregions and therefore more watermarks will be inserted while the accuracy of watermark detection should be enhanced. We thus have the following lemma.

LEMmA 3.1. Given a map $M$ with total length $\mathcal{L}$ and an algorithm threshold $\theta$, the minimum detection confidence for $M$ :

$$
\operatorname{conf}_{\text {min }}(M)=1-\sum_{i=\lceil\rho \mathcal{L} / 4 \theta\rceil}^{\lceil\mathcal{L} / 4 \theta\rceil}\left(\begin{array}{c}
\lceil\mathcal{L} / 4 \theta\rceil \\
i
\end{array}\right)\left(\frac{1}{2}\right)^{\lceil\mathcal{L} / 4 \theta\rceil}
$$

where $\rho$ is the ratio between the number of leaf nodes that match and the total number of leaf nodes in $M$.
PROOF. Straightforward as the total number of leaf nodes is no less than $\mathcal{L} / 4 \theta$.

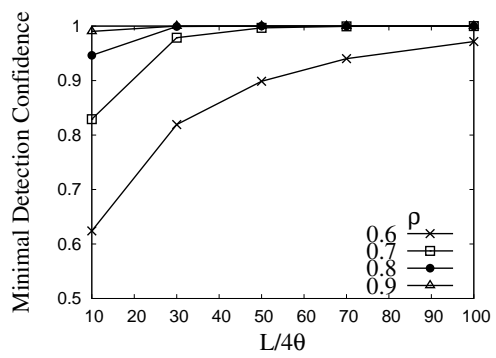

Figure 9: Accuracy of Detection

Figure 9 plots the relationship between minimum detection confidence against $\mathcal{L} / 4 \theta$ for different values of $\rho$, which coincides with our previous intuition. However, smaller $\theta$ translates into more watermarks and hence larger distortion to the map. It also causes the watermarking to be more time-consuming. This trade-off should be carefully considered when applying our approach.

\section{EVALUATION}

In this section, we present some experimental results from the proposed framework.

\subsection{The Experimental Setup}

We implement and test the performance of the algorithms under different potential attacks. All algorithms are implemented with $\mathrm{C}$ and all experiments are conducted on Core PC with $2.0 \mathrm{GHz}$ CPU and 2 GBytes of memory running the Ubuntu 12.04 operating system.

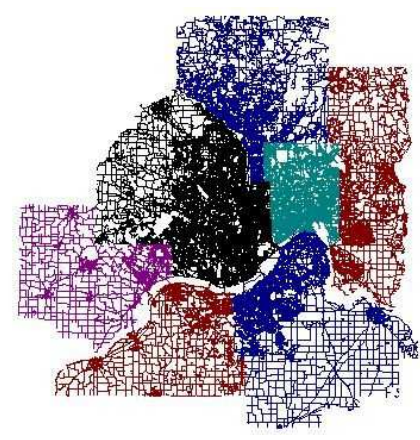

Figure 10: Twin-Cities Seven-County Road Map

We use MN state base map of seven counties, namely, Anoka, Carver, Dakota, Hennepin, Ramsey, Scott, Washington, as our real data set for this experiment. In this data-set, there are in total 415,651 line segments and 372,466 different points. Figure 10 shows the visual map of digital data for these seven counties. This data set can be downloaded from the MN/DOT web site[1].

In order to evaluate the robustness of our watermarking algorithms (which is called Jiang in the rest of this section), we illustrate the performance of our proposed watermark approach under different attacks with different settings. In this part, we compare the proposed approach with two other watermarking algorithms proposed by $\mathrm{Pu}[17]$ and Voigt[23]. Both methods are blind watermarking algorithms and provide some resistance to crop attack. We control 
the accuracy lost of the experiment data caused by different methods and compare the performance of them under noise attack, crop attack and merge attack. In this section, "crop ratio" and "merge ratio" mean the area ratio we crop from the total watermarked map. We set $\xi$ as 0.95 .

\subsection{Performance under Noise Attack}

Noises are added to randomly selected subsets of watermarked maps. Meanwhile, to keep the usability of a map, those subsets could not take too much proportion of the total map. In this experiment, we watermark the map of St. Paul area and attack the watermarked map with some random Gaussian noise, perturbing the map with different accuracy lost.

In this noise attack experiment, we watermark the map with algorithms of $\mathrm{Pu}[17]$, Voigt[23] and Jiang in certain distortion. After that we attack the watermarked maps with different noise distortion. For each noise distortion, the noise will be added in different methods for 20 times. For all three methods, the noise added is "exactly" the same each time. At last, we detect the watermark and calculate detection accuracy for three algorithms. We set distortion $\delta_{w}$ added by the watermarking as $10.5 \times 10^{-6}$ (Jiang), $10.8 \times 10^{-6}$ (Voigt) and $9.8 \times 10^{-6}(\mathrm{Pu})$. Take the size of map into consideration, these distortion can be deemed at the same level. On the other hand, the noise distortion $\delta_{n}$ is changed from $5.12 \times 10^{-3}$ to $2.56 \times 10^{-2}$. Actually, $\delta_{n}$ here is large enough that almost changes all vertexes of the map. Figure 11 shows the results.

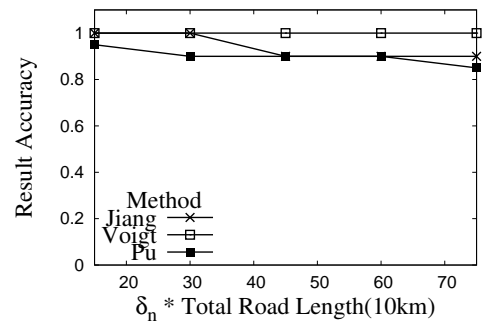

Figure 11: Performance under Noise Attack

In this experiment, we set the standard of positive detection as correctly decide the whole map is watermarked. However, in fact, according to the detection method of our algorithm, some more assistance can be obtained. Even the whole map is failed to be detected, the result could be a series of sub-regions that is suspicious. The results show that our algorithm successfully survived noise attack. On one hand, we watermark certain bits which will not be directly affected by the noise if perturbing for individual vertex is under certain strength. Meanwhile, even the perturbing for individual vertex is powerful enough, $\theta$ and the hash function of $s l$ in in partition and insertion algorithms provide a good tolerance for it.

\subsection{Performance under Crop Attack}

In our experiment, on one hand, each county out of seven is selected to be detected. On the other hand, some certain proportion of the map is selected to evaluate the performance of our algorithm.

In this experiment, the parameter $\theta$ is set as 30,000.0 $\left(\delta_{w}=\right.$ $10.5 \times 10^{-6}$ ) and the square side length is 100.0 for both insertion and detection. We partition the whole map of Minneapolis-St. Paul Metropolitan area. Then the watermarked map is split according to the boundary of different counties. After that, we impose our watermarking algorithm on these "partial" maps, trying to decide whether the maps are parts of our original map. Table 2 shows the results of this experiment.
Table 2: Crop Attack Detection

\begin{tabular}{|c||c|c|c|c|}
\hline County & Total & Match & Confidence & Result \\
\hline \hline Anoka & 55 & 51 & 1.000 & positive \\
\hline Carver & 28 & 23 & 0.999 & positive \\
\hline Dakota & 68 & 61 & 1.000 & positive \\
\hline Hennipin & 141 & 137 & 1.000 & positive \\
\hline Ramsey & 58 & 56 & 1.000 & positive \\
\hline Scott & 29 & 25 & 0.999 & positive \\
\hline Washington & 46 & 43 & 1.000 & positive \\
\hline
\end{tabular}

By analyzing the insertion algorithm, we can easily get the notion that the partition criterion $\theta$ has a large influence on the result of our watermark algorithm. We also design a series of experiments to figure out the influence of the $\theta$. In this experiment, we watermark the map with different distortion (2.1) by adjusting $\theta$ (see Figure 13(a)) and then select the crop ratio of $1 / 2,1 / 4$ and $1 / 8$ of the map to detect. Each ratio is detected for 20 times. Figure 13(b) show the result of experiments. Insertion (Figure 13(c)) and detection (Figure 13(d)) time for different distortion is also measured.
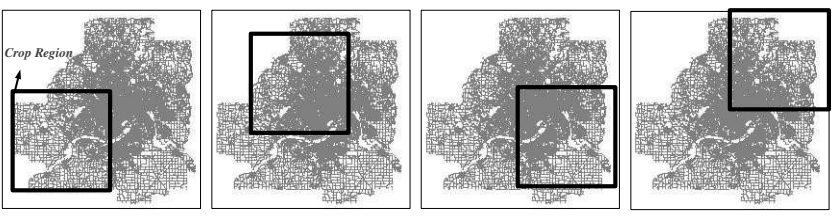

Figure 12: A Showcase of Crop Ratio 1/4

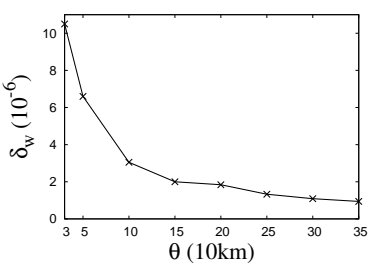

(a) Distortion

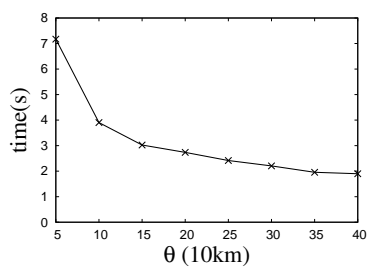

(c) Time of Insertion

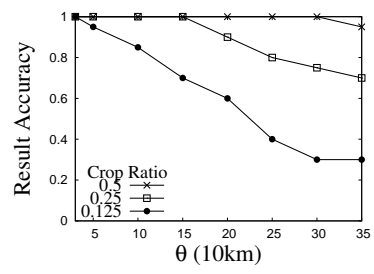

(b) Different Crop Ratio

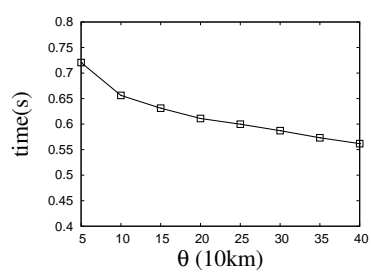

(d) Time of Detection

\section{Figure 13: Experiment Results under Different Distortion}

In the following experiment, we watermark a map with certain distortion, here we set $\theta=30,000$. After that we crop different ratio from the map to evaluate the performance of our algorithm. For each crop ratio, we select different parts of the map to detect up to 20 times. We randomly select different parts of the watermarked map to detect (see Figure 12) and calculate the detection accuracy. Here we also implement Voigt's and Pu's methods to make a comparison. Distortion $\delta_{w}$ added by the watermarking is $10.5 \times 10^{-6}$ (Jiang), $10.8 \times 10^{-6}$ (Voigt) and $9.8 \times 10^{-6}(\mathrm{Pu})$. The distortion of all three methods are almost at the same level. Figure 14(a) gives the results. 


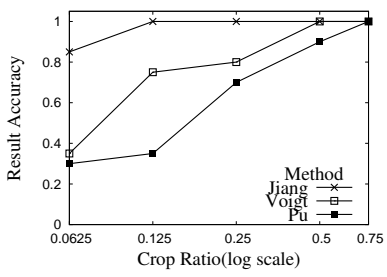

(a) Performance under Cropping

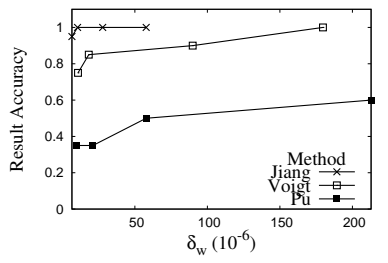

(b) Accuracy in $1 / 8$ crop ratio
Figure 14: Performance under Crop Attack

According to the experiment result, we can find that our algorithm keeps a perfect result when the crop ratio is up to $1 / 8$, while the comparison methods show a significant accuracy degradation. And when crop ratio is $1 / 16$, we still contain a high accuracy. We also test the detection accuracy under different distortion in $1 / 8$ crop ratio (see Figure 14(b)). In this experiment, our algorithm can attain a $100 \%$ detection accuracy with just little distortion. Since the detection accuracy of our algorithm quickly attain to 1 , it is not necessary to plot too many points in Figure 14(b).

\subsection{Performance under Merge Attack}

In this experiment, we select the 2012 TIGER map of MinneapolisSt. Paul Metropolitan area as another data source, which can be downloaded from the United States Census Website[2]. The coordinate system of TIGER map is different from MN dot base Map. We transform the coordinate system of TIGER map to make it same as the former data source. Figure 15 is the overlap of these two maps of Anoka county after the synchronization.

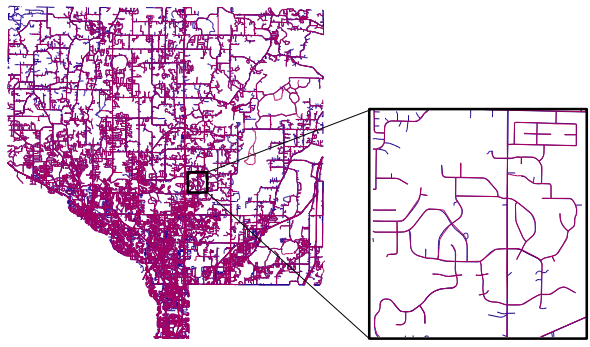

Figure 15: Overlap of Two Maps

We crop a watermarked map $\left(\delta_{w}=6.60 \times 10^{-6}\right)$ and merge part of it with TIGER map to make a "new" map (see Figure 16(a)). The result of the merge detection: suspicious regions and detection confidence is shown in Table 3. Here we select those regions which have at least 10 points tested. From the corresponding visual figure (see Figure 16(b)), we can see that our algorithm almost "exactly" decides the suspicious regions.

Table 3: Merge Attack Detection

\begin{tabular}{|c||c|c|c|c|}
\hline Region & Total & Match & Confidence & Result \\
\hline \hline 1 & 147 & 122 & 1.000 & positive \\
\hline 2 & 12 & 11 & 0.997 & positive \\
\hline 3 & 23 & 18 & 0.994 & positive \\
\hline 4 & 15 & 12 & 0.983 & positive \\
\hline
\end{tabular}

We also watermark the Minneapolis-St. Paul Metropolitan area and then split this watermarked map into the same small portions as cropping experiment above. After that we merge these maps with

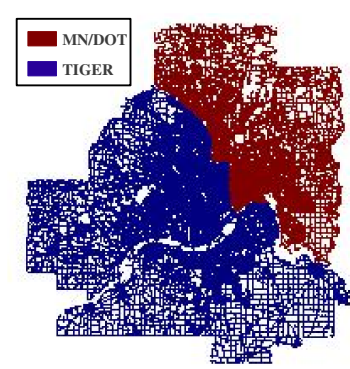

(a) Merge Attack

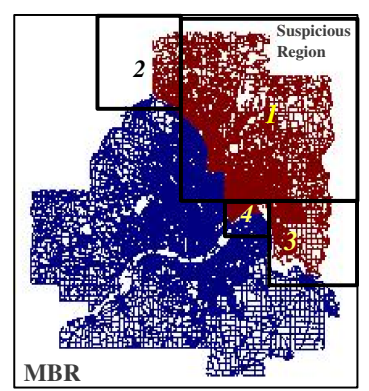

(b) Detection Result
Figure 16: Merged Map

the complementary parts of map from TIGER to create some new digital road maps. In this experiment, we also implement the two other method to make a comparison. Distortion $\delta_{w}$ added by the watermarking still is $10.5 \times 10^{-6}$ (Jiang), $10.8 \times 10^{-6}$ (Voigt) and $9.8 \times 10^{-6}(\mathrm{Pu})$. Figure 17(a) shows the result of our experiments.

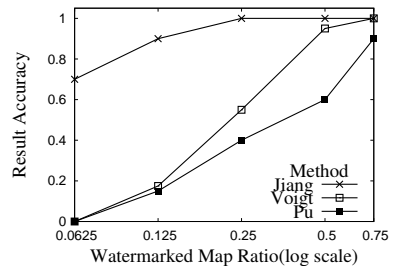

(a) Performance under merging

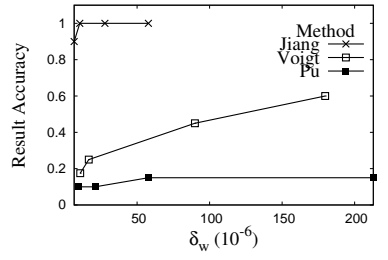

(b) Accuracy in $1 / 8$ merge ratio
Figure 17: Performance under Crop Attack

Comparing with the cropping experiment, we can obviously find the performance of our proposed algorithm is almost not affected by the complementary part of the map. However, the detection accuracy of the other two methods decrease significantly. The reason is the complementary map provides sufficient disturb to watermarked data. Similarly, we also implement another experiment to evaluate the relationship between detection accuracy and watermark distortion $\delta_{w}$. From this figure, we can see that our algorithm still can quickly attain a quite high detection accuracy. However, when $\delta_{w}$ increases for Pu's and Voigt's method, the detection accuracy doesn't changed significantly. The reason for Pu should be that it's global liner correlation is destroyed by "merge" attack. For Voigt's method, though more watermark information is used for detection, more "merge" noise will will also be added to the detection process. Thus increase of detection accuracy for them is not significant.

\section{RELATED WORK}

Many efforts have been made to protect the copyrights of multimedia products such as images, movies and music. Digital road maps is a special vector graphics data which contains important geographical and spatial information. In the rest of this section, we will focus on GIS spatial data watermarking but will also briefly touch on related techniques in some other domains. The technique proposed in this paper benefits from or is inspired by some of these methods.

\subsection{GIS Spatial Data Watermarking}

Several studies have touched on the watermarking of GIS spatial data such as digital vector maps. Even though digital vector maps 
are represented by node coordinates which can also be treated as relational tables, techniques developed for relational data ignore the spatial properties of road maps such as spatial distances and geolocalities. These distinct features of GIS spatial data call for special treatments in watermarking techniques. According to Niu [14], the existing methods of digital vector map watermarking is classified into two categories: algorithms in spatial domain and algorithms in frequency domain. Depending on whether to require the original map for detection, these methods are also classified as the not blind and the blind. In addition, algorithms can also be categorized into global or local depending on whether global information of the map is needed when computing each watermark.

The spatial domain algorithms embed watermarks based on the geometric properties of polyline and polygon objects. It is often easier to control the amount of distortion added by the watermarks. They are also more robust against rotation, scaling and noises, while preserving the utility of a map. Existing methods in spatial domain usually lack of robustness against massive cropping and merging. The method proposed in this paper falls into the category of spatial domain algorithms.

The frequency domain algorithms essentially transform the original data into frequency domain, usually by Discrete Fourier Transform (DFT) [11, 21, 22] or Discrete Wavelet Transform (DWT) [13], add noises in the frequency domain and then transform it back into the spatial domain. The critical drawbacks of frequency domain techniques are the difficulty of controlling the amount of distortion in the spatial domain and the lack of robustness against data cropping and data reordering, due to the fact that it is actually a global watermarking scheme.

\subsubsection{Non-Blind Methods in Spatial Domain}

Some watermarking methods $[7,15,16]$ require the original map or watermarked map as a reference to detect watermarks from a suspicious map. This kind of methods are called "non-blind" algorithms. The main weakness of these methods is that road maps can be updated over time and a map producer may have many versions of the same map in its database. Moreover in the same database, there could be many maps which have overlapping regions, say map of the Twin Cities, map of Minnesota, map of the United States, etc. When the map database is big, and when the suspicious map could have been subjected to cropping and merging, it is not easy to identify the original map.

Ohbuchi et al.[15] partitions the space of the digital map into rectangles such that every rectangle contains almost equal node numbers. Then the $v^{t h}$ vertex inside the rectangle is modified to include a watermark. In another method [16], all vertices are connected into a single mesh by Delaunay triangulation. Then a mesh Laplacian is formed and the mesh is partitioned into patches using the same method as in the former one[15]. Mesh-spectral coefficients are calculated for every patch and the watermark is embedded into these coefficients.

Both of the above algorithms disperse watermarks locally and have some robustness against crop attack. However, them are highly dependent on the validity of the original data. When extracting the watermark, the suspicious map is aligned onto the original map. All inserted nodes have to be deleted, and all removed nodes have to be correctly recovered.

\subsubsection{Blind Methods in Spatial Domain}

Some other watermark framework are proposed which required no original or watermarked map as a reference. Some of these methods $[8,9,24,12]$ insert watermark globally. Yan et al. proposed a key point based algorithm [24]. Key points are those nodes in the map which have more important geometric aspects than the other ones, for example cross joints and those in sharp curve. They are not likely to be removed or edited by the attackers because removing them will render the map useless. This method uses a dynamic programming algorithm [6] both in insertion and detection steps. Key points in the map are used for inserting and detecting watermarks. This kind of algorithms are robust against some attacks like noise and vertex simplification, but are still vulnerable to crop and merge attacks.

Other blind watermarking algorithms $[23,18,17,5,10]$ provide some limited resistance against crop attacks to some extent. Voigt et al.[23] proposed a feature based watermarking algorithm which is relies on statistical detection. This method partitions a map into small rectangular regions called "patches". It then randomly selects two subsets of the patches called set $A$ and set $B$, respectively. Next, it calculates a reference point for each patch in the two set. During watermark insertion, all nodes in set $A$ are shifted toward the reference point in their respective patches, while all nodes in set $B$ are shifted away from the reference point. The amount of shift is governed by an $F$-distribution [3] which is also used to detect the watermark. Pu et al. proposed a blind algorithm [17] which divides the map into mesh segments, and then embeds the watermark in each segment with a fixed order. In the detection step, each segment is evaluated by a correlation parameter, which is a linear correlation of the watermark and the watermarked data. To survive crop attack, a global correlation based detection method is applied as an optimization. This algorithm watermarks maps according to their topological relations.

While these algorithms present some resistance against cropping attack, they cannot survive cropping at larger scale. Take Voigt[23] as an example, when the watermarked map is massively cropped, many patches that are marked may be removed. Thus $F$ distribution of watermarked points may be destroyed. Therefore, the method is not robust enough against "massive crop". Furthermore, if the map is cropped and then merged with others, the information added by the merge will defeat the watermarking more easily.

\subsubsection{Methods in Frequency Domain}

Solachidis et al.[21] proposed a blind watermarking scheme embedding a single bit into a polyline by modifying the discrete Fourier coefficients of polyline's coordinate sequence. This method embeds the watermark in the magnitude of the curve's Fourier descriptors to exploit its location, scale, and rotation invariant properties. Due to the amplitude frequency features of discrete Fourier transform, the algorithm is inherently robust to many attacks such as map translation, rotation, scaling and changing start vertex. Similar to the DFT method[21], Li et al. proposed a blind scheme [13] embedding multi-bits into a vector map in DWT domain. These frequency domain methods rely on the integrity of map and order of data points. When part of map data is removed or the order of data points is changed, the coefficients will also be changed, which makes them extremely vulnerable to crop and merge attacks.

\subsection{Watermarking in Other Domains}

Some research attention has been given to relational data[4, 20]. Rakesh Agrawal proposes a framework of watermarking relational databases[4]. According to this approach, a primary key is stored in some significant tuples of relational data. The altered attribute index and bit index for the selected attribute are randomly selected according to secret keys. Their approach is highly dependent on the primary key of the data tuple and randomly selects a subset of tuples to watermark. This approach in fact disperses one bit watermark into different position of relational data. It provide some 
resistance against crop and merge attack to some extent. Some ideas in this framework actually inspired the work in this paper, e.g., modifying least significant bits and computing the confidence of detection. However, this framework is for relational data and hence does not consider the special properties of spatial attributes in GIS spatial data. In our method, information which is adjacent to each other in geographical space collaborate to decide the watermarking positions. However, in relational data, different tuples are completely independent to each other, even though they are stored together, because they form a set. Other work[20] extends the framework [19] to relational databases. In that paper, numeric dataset are first hashed to another dataset with a secret order. Then the new dataset is grouped into different chunks according to their order. Finally, the average value and standard deviation is calculated for every chunk. According to these statistics and watermarking data, a small number of data points are altered. This approach still disperses the watermark globally. One of the serious drawbacks for this global watermarking approach is that it completely fails a "massive crop" attack.

\section{CONCLUSION}

In this paper, we proposed a new blind watermarking scheme for digital vector road maps. The scheme produces and detects watermarks according to local information with the help of three secret keys of negligible sizes but without referring to the original map. The algorithm dynamically partitions a given map according to road density and inserts one-bit watermarks to one of the least significant bits of points determined by the secret keys. The amount of distortion brought by watermarks is arguably much smaller than existing methods. Our preliminary evaluation shows that this algorithm is resilient to massive crop and merge attacks and significantly outperforms two other state-of-the-art vector map watermarking approaches in terms of detection accuracy.

\section{REFERENCES}

[1] MN/DOT. http://www.dot.state.mn.us/.

[2] TIGER. http://www. census.gov/.

[3] M. Abramowitz and I. Stegun. Handbook of mathematical functions: with formulas, graphs, and mathematical tables, volume 55. Dover publications, 1965.

[4] R. Agrawal and J. Kiernan. Watermarking relational databases. In $V L D B$, pages 155-166, 2002.

[5] S. Bird, C. Bellman, and R. v. Schyndel. A shape-based vector watermark for digital mapping. In Proceedings of the 2009 Digital Image Computing: Techniques and Applications, DICTA '09, pages 454-461, Washington, DC, USA, 2009. IEEE Computer Society.

[6] D. H. Douglas. Algorithms for the reduction of the number of points required to represent a line or its a caricature. The Canadian Cartographer, 10(2):112-122, 1973.

[7] P. Han, J. Gong, and L. Cheng. An improved adaptive watermarking algorithm for vector digital maps. In Geoscience and Remote Sensing Symposium, 2006. IGARSS 2006. IEEE International Conference on, pages $2844-2847$, 31 2006-aug. 42006.

[8] X.-J. Huo, T.-Y. Seung, B.-J. Jang, S.-H. Lee, and K.-R. Kwon. A watermarking scheme using polyline and polygon characteristic of shapefile. In Intelligent Networks and Intelligent Systems (ICINIS), 2010 3rd International Conference on, pages 649 -652, nov. 2010.

[9] H. Kang, K. Kim, and J. Choi. A vector watermarking using the generalized square mask. In ITCC, pages 234-236. IEEE Computer Society, 2001.

[10] J. Kim, S. Won, W. Zeng, and S. Park. Copyright protection of vector map using digital watermarking in the spatial domain. In Digital Content, Multimedia Technology and its Applications (IDCTA), 2011 7th International Conference on, pages $154-159$, aug. 2011.

[11] I. Kitamura, S. Kanai, and T. Kishinami. Copyright protection of vector map using digital watermarking method based on discrete fourier transform. In Geoscience and Remote Sensing Symposium, 2001. IGARSS '01. IEEE 2001 International, volume 3, pages 1191 -1193 vol.3, 2001.

[12] S.-H. Lee and K.-R. Kwon. Vector watermarking scheme for GIS vector map management. DOI10.1007/s11042-011-0894-y SpringerLink, 2011.

[13] Y. Li and L. Xu. A blind watermarking of vector graphics images. In ICCIMA '03: Proceedings of the 5th International Conference on Computational Intelligence and Multimedia Applications, page 424, Washington, DC, USA, 2003. IEEE Computer Society.

[14] X. Niu, C. Shao, and X. Wang. A survey of digital vector map watermarking. In 2006 International Journal of Innovative Computing,Information and Control Volume 2, 2006.

[15] R. Ohbuchi, H. Ueda, and S. Endoh. Robust watermarking of vector digital maps. In ICME (1), pages 577-580, 2002.

[16] R. Ohbuchi, H. Ueda, and S. Endoh. Watermarking 2d vector maps in the mesh-spectral domain. In Shape Modeling International, pages 216-228, 2003.

[17] Y.-C. Pu, W.-C. Du, and I.-C. Jou. Toward blind robust watermarking of vector maps. In ICPR (3), pages 930-933, 2006.

[18] G. Schulz and M. Voigt. A high capacity watermarking system for digital maps. In MM\&Sec, pages 180-186, 2004.

[19] R. Sion, M. J. Atallah, and S. Prabhakar. On watermarking numeric sets. In $I W D W$, pages 130-146, 2002.

[20] R. Sion, M. J. Atallah, and S. Prabhakar. Rights protection for relational data. In SIGMOD Conference, pages 98-109, 2003.

[21] V. Solachidis and I. Pitas. Watermarking polygonal lines using fourier descriptors. Computer Graphics and Applications, IEEE, 24(3):44 - 51, may-jun 2004.

[22] H. Sonnet, T. Isenberg, J. Dittmann, and T. Strothotte. Illustration watermarks for vector graphics. Computer Graphics and Applications, Pacific Conference on, 0:73, 2003.

[23] M. Voigt and C. Busch. Feature-based watermarking of 2d-vector data. In SPIE,Security and Watermarking of Multimedia Content.Santa Clara, pages 359-366, 2003.

[24] H. Yan, J. Li, and H. Wen. A key points-based blind watermarking approach for vector geo-spatial data. Computers, Environment and Urban Systems, 35(6):485-492, 2011. 